\title{
Krisensymptome supranationaler Leitbilder - zur Notwendigkeit intergouvernementaler Integration
}

von Frank Schorkopf

\begin{abstract}
Mit diesem Beitrag soll die These begründet werden, dass die Ursachen der Krise der Europäischen Union sich nicht in der Fehlfunktion einiger Regeln und Institutionen, die vor zwanzig Jahren entworfen und beschlossen wurden, erschöpfen. Sie reichen zeitlich weiter in die Integrationsgeschichte zurück. Eine wesentliche Krisenursache ist der politische Dissens über Intensität und Richtung der europäischen Integration. Das Unionsrecht ist - auch nach seiner Ertüchtigung durch Rettungsfonds, Sechser- und Zweierpakete, durch Semester, Verfahren und Kennziffern - nur begrenzt geeignet, den Konflikt zu lösen. Die Folgebereitschaft der Mitgliedstaaten ist nicht in jedem Fall gegeben, und das Handeln der Unionsorgane hat eine Durchsetzungsschwäche offengelegt. Notwendig ist eine Debatte über die Wirtschaftspolitik in der Union. Die Europapolitik und die sie tragenden gesellschaftlichen Kräfte sollten ihr romantisches Integrationsverständnis endgültig aufgeben oder stärker an realpolitischen Bedingungen eines ,europäischen Deutschlands“ ausrichten. Sechs kürzere Analysen sollen die These begründen, im Sinne eines Zwischenrufs zur europäischen Krise, die bereits eine Legislaturperiode andauert.
\end{abstract}

This contribution argues that the causes of the European crisis should not only be sought in the malfunctioning of a particular set of rules and institutions that were created twenty years ago. Instead, a closer look at the history of the EU appears necessary. One major factor is the on-going political disagreement on the intensity and direction of European integration. EU law is only partially capable of resolving this conflict, despite its recent expansion through rescue funds, six-and two-packs, semesters, further procedures and benchmarks. The willingness of the Member States to adhere to European law is not uniform, exposing a deeply rooted inability of the Union's organs to implement their decisions and triggering a wider debate on European economic policy. Germany's political actors and the social forces that support them should finally abandon their romantic ideal of integration and instead accept the Realpolitik-based condition of a "European Germany". Six short analyses are presented to support this hypothesis, summarising insights drawn from a European crisis that has already spanned an entire electoral term. 


\section{Geldpolitik und die Unabhängigkeit von Zentralbanken}

Vor einiger Zeit berichtete der ehemalige Präsident der Europäischen Zentralbank, Jean-Claude Trichet, in einer Festrede, wie es zur Sitzordnung im EZBRat kam. Die Ordnung, die für das erste Ratstreffen vorbereitet wurde, folgte dem Herkunftsland der Präsidenten der nationalen Zentralbanken. Daraufhin habe Bundesbankpräsident Hans Tietmeyer vorgeschlagen, dass stattdessen die alphabetische Reihenfolge der Namen vorgeben sollte, wer neben wem sitzt. Schließlich seien die Mitglieder des EZB-Rates Vertreter des gesamten EuroRaums und nicht ihrer Mitgliedstaaten. ${ }^{1}$

Die Anekdote ist eine Fußnote. Sie ist aber zugleich beredt, weil sie über Vorverständnisse, Erwartungen und Leitbilder der europäischen Integration Auskunft gibt. Diese handlungsleitenden Motive können, sind sie auf Dauer kontrafaktisch, erhebliche negative Folgen haben. Es besteht kein Zweifel daran, dass der Bundesbankpräsident Recht hatte. Die Unabhängigkeit einer Zentralbank rechtfertigt sich dadurch, dass die für das Primärziel Preisstabilität notwendigen geldpolitischen Maßnahmen von Fachleuten nach Sachkriterien ergriffen werden. Geldpolitik wird erkannt, Fiskalpolitik wird gemacht.

Vom entgegengesetzten Standpunkt aus besteht aber auch kein Zweifel daran, dass er Unrecht hatte. Denn die Praxis macht, vergleichbar mit den allein auf das Unionsinteresse verpflichteten Mitgliedern der Europäischen Kommission, eine weitere Dimension föderaler Willensbildung sichtbar. Für die Glaubwürdigkeit und Akzeptanz geldpolitischer Maßnahmen - und deren Risiken - spielt es eine Rolle, ob Beschlüsse ohne Beteiligung oder stets gegen die Stimmen derjenigen Mitglieder gefasst werden, die die Zentralbanken mit den größten Kapitalanteilen und entsprechend kräftiger Volkswirtschaft wie gewichtigem Finanzsektor vertreten.

Die erhebliche Aufmerksamkeit, die die Bundesbank für ihre Haltung zu jüngeren EZB-Maßnahmen erhält, ${ }^{2}$ beruht nicht allein auf dem hohen Ansehen einer

1 Trichet, J.-C.: Laudatio für Hans Tietmeyer im Rathaus der Stadt Münster, 26.3.2010, abrufbar unter www.ecb.int. Diese Praxis der Sitzordnung hat auch die Auslegung der einschlägigen Normen für den Status der Mitglieder des EZB-Rates geprägt, s. Art. 10.2 ESZB-/EZB-Satzung, wonach die Mitglieder des EZB-Rates ihr Amt persönlich wahrnehmen müssen, eine Stellvertretung ausgeschlossen ist und nur bei längerfristiger Verhinderung ein Stellvertreter vom Mitglied benannt werden kann. Tietmeyer bestätigt diese Anekdote in seinen Erinnerungen, ders.: Herausforderung Euro, 2005, 268.

2 Siehe vor allem die Reaktionen auf die Stellungnahme der Deutschen Bundesbank v. 21.12.2012 gegenüber dem Bundesverfassungsgericht in dem Hauptsacheverfahren 2 BvR 1390/12 u.a., die das Handelsblatt öffentlich gemacht hat. 
über Zweifel erhabenen, mit dem Erfolg der D-Mark verbundenen Institution. Sie rührt zumindest auch daher, dass im Rahmen öffentlicher Äußerungen von Zentralbankern bekannt geworden ist, der Präsident der Bundesbank habe die Beschlüsse über die Programme für Staatsanleihekäufe als einziges Mitglied des EZB-Rates abgelehnt. ${ }^{3}$ Die ohnehin kritische Grundhaltung in der deutschen Öffentlichkeit zu einzelnen Krisenmaßnahmen wird so durch den Eindruck verstärkt, ,die anderen“ beschlössen finanzwirksame Maßnahmen gegen den deutschen Willen, obwohl Deutschland garantiere, bürge und hafte.

In der öffentlichen Debatte ist dabei aus dem Blick geraten, dass die Rotationsregeln über die Willensbildung im EZB-Rat solche Beschlüsse sogar ohne Stimmbeteiligung des deutschen Mitglieds ermöglichen. Ab dem Zeitpunkt, in dem mehr als 15 Präsidenten nationaler Zentralbanken im EZB-Rat sitzen, werden die Präsidenten in zwei, später in drei Gruppen je nach Wirtschaftskraft und Relevanz des Finanzsektors ihrer Mitgliedstaaten eingeteilt. Die Gruppen unterscheiden sich danach, wie häufig ihre Mitglieder stimmberechtigt sind. ${ }^{4}$ Der Bundesbankpräsident würde der ersten Gruppe angehören, in der fünf Mitglieder sich vier Stimmrechte teilen, d.h. das deutsche Mitglied wäre jeden fünften Monat nicht stimmberechtigt. Das Anwesenheits- und Rederecht ist von der Rotationsregel ausgenommen.

Der EZB-Rat hat zwar im Jahr 2008 anlässlich der Euro-Einführung in der Slowakei beschlossen, die Rotationsregel erst anzuwenden, wenn die Anzahl der Präsidenten der nationalen Zentralbanken im EZB-Rat 18 überschreitet. ${ }^{5}$ Doch wie hätte die öffentliche Debatte ausgesehen, wenn die Ankündigung von Outright Monetary Transactions (OMT) ${ }^{6}$ ohne Beteiligung des deutschen Mitglieds im EZB-Rat gefasst worden wäre? Das Rotationssystem ist erst durch den Vertrag von Nizza, der 2003 in Kraft trat, in das Primärrecht gelangt. Der Vertrag fügte in die Satzung eine Ermächtigung zur vereinfachten Vertragsänderung ein, ${ }^{7}$ die einen ratifikationsbedürftigen Beschluss des Europäischen Rates vorsah.

3 Zuletzt Weidmann, J.: „Nicht alles ist erlaubt“, Interview mit der Süddeutschen Zeitung v. 24.6.2013, Nr. 143, 18.

4 Art. 10.2 EZSB-/EZB-Satzung, Art. 3a GO-EZB; zu den Einzelheiten Steven, C.: Art. 10 Satzung, in: Siekmann, H. (Hg.), Europäische Währungsunion, 2013, Rn. 5 ff.

5 Europäische Zentralbank: Beschluss v. 18.12.2008, EZB/2008/29, AB1. 2009 L 3/4. Im Zwei-GruppenRotationssystem hätte die Stimmrechtsberechtigung der zweiten Gruppe, der kleineren EuroMitgliedstaaten, bei 100\% gelegen, während es in der ersten Gruppe zur Rotation gekommen wäre.

6 Europäische Zentralbank: Technical Features of Outright Monetary Transactions, Presseerklärung v. 6.9.2012; Monatsbericht, September 2012, 7-12.

7 Art. 10.6, eingefügt durch Art. 5 des Vertrages von Nizza, ABl. 2001 C 80/42. 
Das Rotationssystem wurde kurz vor Aufnahme der zehn Transformationsstaaten in die Union beschlossen, um unabhängig von der Anzahl der Mitgliedstaaten, die den Euro einführen, sicherzustellen, ,dass der EZB-Rat weiterhin in der Lage ist, in einem erweiterten Euro-Währungsgebiet Entscheidungen effizient und rechtzeitig zu treffen. “8

Diese Änderung des institutionellen Rahmens war also dem Ziel geschuldet, die Europäische Zentralbank in ihrer Aufgabe zu stärken, unabhängig und sachgerecht das Primärziel der Preisstabilität zu gewährleisten. Zugleich sollte die gerade von Deutschland angestrebte „Osterweiterung“ verwirklicht werden. Nur aufgrund dieser Interessenlage ist erklärbar, dass es im Ratifikationsverfahren zum Rotationsbeschluss weder eine Aussprache im Bundestag noch im Bundesrat gab. Die damals oppositionelle CDU/CSU-Fraktion hatte in einer Entschließung immerhin gefordert, dass möglichst kurze Intervalle, d.h. ein Zeitraum von nicht mehr als drei Monaten beim Stimmrechtswechsel wünschenswert seien, so dass größere Länder fast durchgängig ein Stimmrecht hätten ausüben können. Die regierungstragende Mehrheit mahnte an, dass eine formale Entschließung des Bundestages mit diesem Inhalt wegen der Unabhängigkeit der Europäischen Zentralbank nicht statthaft sei. ${ }^{9}$

Die Regeln für die Willensbildung im EZB-Rat sind solange unproblematisch, als die Überzeugung vorherrscht, dass Geldpolitik durch Sachverstand erkannt wird. Deshalb wird die aktuelle Beschlusslage zu Staatsanleihekäufen mit dem Hauptargument kritisiert, es handele sich nicht mehr um Geld-, sondern um parlamentarisch zu verantwortende Fiskalpolitik. ${ }^{10}$ Was geschieht aber mit diesem

8 1. Erwägungsgrund des Beschlusses des Rates in der Zusammensetzung der Staats- und Regierungschefs v. 21.3.2003 über eine Änderung des Artikels 10.2 der Satzung des Europäischen Systems der Zentralbanken und der Europäischen Zentralbank, AB1. 2003 L 83/66; näher zum Inhalt vgl. die Denkschrift des Auswärtigen Amtes, BR-Drs. 552/03, 7 f.

9 Deutscher Bundestag: Beschlussempfehlung und Bericht des Finanzausschusses, BT-Drs. 15/2008, 5: „Die Koalitionsfraktionen haben jedoch grundsätzliche Bedenken gegenüber einer gesonderten Entschließung des Deutschen Bundestages gegenüber der Europäischen Zentralbank zur Dauer der Intervalle geltend gemacht. Für die Koalitionsfraktionen habe die Unabhängigkeit der Europäischen Zentralbank mit samt ihrer Satzungsautonomie oberste Priorität, weshalb nationale Gesetzgeber, so auch der Deutsche Bundestag, hier zur Zurückhaltung aufgefordert seien.“ Aus der Drucksache geht hervor, dass eine nichtöffentliche Anhörung stattgefunden hat, in der die Bundesbank für die zeitliche Begrenzung der stimmrechtslosen Intervalle eintrat.

10 Für die Einzelheiten Kahl, W.: Die Bewältigung der Staatsschuldenkrise unter Kontrolle des Bundesverfassungsgerichts, in: Deutsches Verwaltungsblatt 128 (2013), 197-207; Siekmann, H./Wieland, V.: The European Central Bank's Outright Monetary Transactions and the Federal Constitutional Court of Germany, Institute for Monetary and Financial Stability, Working Paper Series 71 (2013); Sester, P.: Plädoyer für die Rechtmäßigkeit der EZB-Rettungspolitik, in: Recht der internationalen Wirtschaft 59 (2013), 
kritischen Standpunkt, wenn es sich um geldpolitische Maßnahmen handelt, eine Position, für die die Europäische Zentralbank eine substantielle Begründung anbietet?

Das Argument wird noch deutlicher, wenn wir den bislang wenig beachteten Art. 20 der ESZB-/EZB-Satzung in Blick nehmen. Der EZB-Rat kann mit Zweidrittelmehrheit über ,,andere Instrumente der Geldpolitik“ entscheiden, die aus seiner Sicht unter Beachtung der Ziele zweckmäßig sind. Bereits die qualifizierte Mehrheit ermöglicht ein Handeln auch gegen den deutschen Willen. Der Artikel ist eine instrumentenbezogene „Flexibilitätsklausel“, die im Normprogramm der Wirtschafts- und Währungsunion von Beginn an enthalten ist und die nicht nur vom verfassungsändernden Gesetzgeber ratifiziert wurde, sondern in zwei einschlägigen Verfahren vor dem Bundesverfassungsgericht unbeanstandet blieb. ${ }^{11}$

Die Krise macht sichtbar, dass die Unabhängigkeit von Zentralbanken, die nach deutschem Verfassungsrecht notwendige Bedingung für die gemeinsame Währung ist, ${ }^{12}$ Zumutungen mit sich bringen kann, die bislang nicht gesehen oder erwartet wurden. Das wird deutlich, wenn wir den Europäischen Stabilitätsmechanismus (ESM) mit betrachten. Der völkerrechtliche Vertrag über die Errichtung des ESM war für das Bundesverfassungsgericht nur deshalb mit dem Grundgesetz vereinbar, so legt es die Urteilsbegründung im Eilverfahren nahe, weil die Stimmen im ESM-Gouverneursrat nach Kapitalanteilen gewichtet sind und Deutschland deshalb faktisch ein Veto hat. ${ }^{13}$

Letztendlich ist der geltende Rechtsrahmen von der deutschen Regierung entscheidend mitgeformt und vom Willen des deutschen Gesetzgebers getragen.

451-456; mit breiterem Ansatz Murswiek, D.: Von der Stabilitätsunion zur Haftungs- und Transferunion, in: Festschrift für Rolf Stürner, 2013, 1925-1940.

11 BVerfGE 89, 155 - Maastricht (1993); 98, 350 - Euro-Einführung (1998).

12 Art. 88 Satz 2 GG. In der ökonomischen Literatur wird die Unabhängigkeit von Zentralbanken in neuerer Zeit prinzipiell hinterfragt vgl. Taylor, J. B.: The Effectiveness of Central Bank Independence versus Policy Rules, American Economic Association Meeting, Januar 2013; kritisch zu dieser Entwicklung Issing, O.: A New Paradigm for Monetary Policy?, CFS Working Paper Nr. 2013/02, 11-15.

13 Soweit nicht im Einvernehmen der Vertragsparteien entschieden wird, werden Beschlüsse im Gouverneursrat mit qualifizierter Mehrheit gefasst, für die $80 \%$ der abgegebenen Stimmen erforderlich sind, Art. 4 Abs. 5 ESMV, dadurch wird ein „Zahlungs- und Gewährleistungsautomatismus“ ausgeschlossen vgl. BVerfG, Urt. v. 12.9.2012, 2 BvR 1390/12, 2 BvR 1421/12, 2 BvR 1438/12, 2 BvR 1439/12, 2 BvR 1440/12 und 2 BvE 6/12, abgedruckt in: Neue Juristische Wochenschrift 2012, 3145 ff., Ziff. 279, auch 213; zur Einordnung dieses Urteils in die Rechtsprechung des Zweiten Senats Huber, P. M.: Zum Verständnis des Bundesverfassungsgerichts vom Kompetenzgefüge zwischen EU und den Mitgliedstaaten, in: Möllers/Zeitler (Hg.), Europa als Rechtsgemeinschaft - Währungsunion und Schuldenkrise, 2013, 229-243. 
Musterbeispiele für möglicherweise sachgerechtere Lösungen des nunmehr aufscheinenden Problems wurden nicht aufgegriffen. Das häufig als Rollenvorbild zitierte amerikanische Notenbanksystem kennt auch ein Rotationssystem. Die Zentralbank für New York ist im Offenmarktauschuss (Federal Open Market Committee) gleichwohl stets stimmberechtigt ${ }^{14}$ - wie könnte die für die Wall Street zuständige Institution auch bei geldpolitischen Entscheidungen fehlen. Die deutsche Europapolitik und die sie tragenden gesellschaftlichen Kräfte haben Wechsel ausgestellt, die nun eingelöst werden. Sie haben ihr Integrationsverständnis zu stark an idealisierten Leitbildern statt an realpolitischen Bedingungen ausgerichtet.

\section{Recht und Politik: Die Durchsetzung der wirtschafts- und haushaltspolitischen Überwachung}

Seitdem die Staatsschuldenkrise im Oktober 2009 begann, als die seinerzeit neu gewählte griechische Regierung das Defizit bei der Haushaltsfinanzierung von $3,7 \%$ auf zunächst $12,7 \%$ korrigierte, ${ }^{15}$ haben sich vier Handlungsfelder gebildet, in denen das Unionsrecht krisenbedingt teilweise erheblich verändert wurde: die fiskalische Sicherung der Euro-Staaten gegen Insolvenz durch Rettungsfonds, die Regulierung des Finanzmarktes unter dem Stichwort der „Bankenunion“, die europäische Haushaltsüberwachung der Mitgliedstaaten und die Koordinierung der nationalen Wirtschaftspolitiken.

Während die wirtschafts- und währungsbezogenen Rechtsetzungsaktivitäten der Union und der Mitgliedstaaten in den letzten drei Jahren eine Tatsache sind, werden die Bedeutung des Rechts in der Union und die Notwendigkeit rechtlicher Handlungsformen unterschiedlich bewertet. Der neue Vertragsartikel etwa, mit dem das Unionsrecht eine normative Schnittstelle zur völkervertraglich organisierten Finanzhilfekapazität, wie dem Europäischen Stabilitätsmechanismus, erhalten hat, ist nach amtlichem Standpunkt deklaratorisch. Der Europäische Gerichtshof hat in seinem Urteil in der Rs. Pringle festgestellt, dass Art. 136 Abs. 3 AEUV die mitgliedstaatliche Kompetenz für die Errichtung eines Stabilitätsmechanismus lediglich bestätige. Die Änderung schaffe keine Rechtsgrund-

14 Section 12A Federal Reserve Act; 12 United States Code 263(a).

15 Europäische Kommission: Bericht zu den Statistiken Griechenlands über das Öffentliche Defizit und den Öffentlichen Schuldenstand, KOM (2010) 1 v. 8.1.2010; für einen Versuch, die Korrektur der Verwaltungsmängel kritisch zu begleiten, Europäischer Rechnungshof, Sonderbericht Nr. 12/2012 v. 21.9.2012: „Wurden die Verfahren zur Erstellung zuverlässiger und glaubwürdiger europäischer Statistiken von der Kommission und Eurostat verbessert?" 
lage, die es der Union ermögliche, eine Handlung vorzunehmen, die vor dem Inkrafttreten der Änderung des AEU-Vertrages nicht möglich gewesen wäre. ${ }^{16}$ Obwohl diese Rechtsansicht von der Bundesregierung geteilt wird, ist der Vertragsartikel, der immerhin der Ratifikation durch die 27 EU-Mitgliedstaaten bedurfte, auf maßgeblich deutsches Betreiben in das Primärrecht aufgenommen worden.

Mit besonderer Empfindsamkeit wird in Deutschland wahrgenommen, dass die Krise ein unterschiedliches kulturelles Verständnis der Institution Recht offengelegt hat. Der Selbststand des Rechts wird allgemein akzeptiert, soweit Recht als handwerkliche Ressource eingesetzt wird, um politische Entscheidungen für den administrativen Vollzug zu reformulieren. Die substantielle Dimension des Rechts als eine der Politik maßstabsetzende und den politischen Gestaltungswillen begrenzende Ressource, wird nicht in allen Mitgliedstaaten geteilt. ${ }^{17}$ Es ist der Eindruck entstanden, die - vom deutschen Kommissionspräsidenten Walter Hallstein gestiftete - Idee der Rechtsgemeinschaft sei nicht der fraglos akzeptierte Knotenpunkt, an dem die Fäden zusammengeführt sind.

Allerdings ist nicht jede Auslegung des Unionsrechts, die von anderen abgelehnt wird, gleichbedeutend mit einer Verneinung des Rechtsgedankens in der Union. Der Europäische Gerichtshof hat mit seiner Auslegung der „Nichtbeistandsklausel“ (Art. 125 AEUV) in der erwähnten Rs. Pringle sicherlich einen Weg eingeschlagen, der eine strenge Sicht auf das Gebot der eigenverantwortlichen Haushaltsfinanzierung dämpft. ${ }^{18}$ Die Auslegung, Art. 125 AEUV verbiete es nicht, anderen Mitgliedstaaten freiwillig eine Finanzhilfe zu gewähren, vorausgesetzt, Konditionalität eigne sich, diesen zu einer soliden Haushaltspolitik zu bewegen, ist gleichwohl vertretbar. Sie wurde sogar mit einem Argument aus der Entstehungsgeschichte des Primärrechts begründet. ${ }^{19}$ So entzündet sich die Kritik deshalb wohl eher an der praktischen Einsicht, dass das letzte Wort zur verbindli-

16 EuGH (Plenum), Rs. C-370/12, Pringle, Urt. v. 27.11.2012, Rn. 72 f., abgedruckt in: Neue Zeitschrift für Verwaltungsrecht 2013, 49 (53).

17 Dazu näher Schorkopf, F.: Gestaltung mit Recht, in: Archiv des öffentlichen Rechts 136 (2011), 323344.

18 EuGH, op. cit. (Fn. 16), Rn. 129-147; bestätigend de Gregorio Merino, A.: Legal Developments in the Economic and Monetary Union During the Debt Crisis: The Mechanisms of Financial Assistance, in: Common Market Law Review 49 (2012), 1613. Vom entgegen gesetzten Standpunkt wird angemerkt, der EuGH seit weit gegangen, indem er politische Stabilität als Ziel für freiwillige Finanzhilfen unter Art. 125 AEUV ausgeschlossen habe, vgl. Borger, V.: The Debt Crisis and Development of Solidarity, in: European Constitutional Law Review 9 (2013), 7 (23 ff., 34 f.).

19 EuGH, op. cit. (Fn. 16), Rn. 135. 
chen Auslegung existenzwichtiger Rechtsnormen auf einen überstaatlichen Spruchkörper übertragen wurde. Im Ergebnis stützt der Gerichtshof im Übrigen die von Bundestag und Bundesregierung eingeschlagene politische Linie.

Die Bedeutung des Rechts in der Europäischen Union ist weiterhin hoch. Der Fiskalvertrag, mit dem die Mitgliedstaaten ihre Haushaltsdisziplin wechselseitig fördern und die nationalen Wirtschaftspolitiken besser aufeinander abstimmen wollen, ${ }^{20}$ drückt dieses Vertrauen in das Unionsrecht aus. Denn der Fiskalvertrag ist aus dem Gedanken entstanden, die sekundärrechtlichen Verpflichtungen der Mitgliedstaaten im Bereich Haushaltsdisziplin auf die Ebene des Primärrechts zu bringen. Dieser Versuch einer Konstitutionalisierung des Stabilitäts- und Wachstumspaktes, mit dem die mitgliedstaatliche Regeltreue verbessert werden soll, ist im Ergebnis zwar nur teilweise geglückt; Großbritannien und Tschechien waren nicht zu einer Primärrechtsänderung bereit, so dass 25 Mitgliedstaaten auf die intergouvernementale Methode auswichen und einen völkerrechtlichen Vertrag schlossen. ${ }^{21}$ Das Vorhaben des Fiskalvertrages zeigt jedoch, dass die verantwortlichen Personen und Einrichtungen, die ihn konzipierten und durchsetzten, an das Recht glauben. Sie trauen dem Vertrag zu, die erwartete Steuerungsleistung zu erbringen.

Die europäische Grenzlinie zwischen Recht und Politik verläuft allerdings stärker zugunsten politischen Handelns, als das aus dem nationalen Kontext vertraut ist. Es ist nicht ausgemacht, wie das Ringen um den Grenzverlauf letztlich ausgehen wird. Die Entwicklungen im Bereich der Haushaltsüberwachung deuten an, dass es zu einem formalen Kompromiss kommen könnte, d.h. eine weitere Verrechtlichung der Wirtschafts- und Währungsunion bei zeitgleicher Politisierung der Rechtsdurchsetzung. Die ertüchtigten Regeln für die wirtschafts- und haushaltspolitische Überwachung sind dafür das Beispiel.

Im Dezember 2011 traten sechs Sekundärrechtsakte, das sog. Six Pack in Kraft, das den Rahmen für die Wirtschafts- und Haushaltspolitik erheblich verändert

20 Vertrag über Stabilität, Koordinierung und Steuerung in der Wirtschafts- und Währungsunion v. 2.3.2012, BGB1. II 2012, 1006, der Vertrag ist am 1.1.2013 in Kraft getreten, Bek. v. 14.1.2013, BGB1. II 2013, 162. Näher zum Fiskalvertrag Europäische Zentralbank, Monatsbericht 5/2012, 85-102; Craig, P.: The Stability, Cordination und Governance Treaty: Principle, Politics and Pragmatism, in: European Law Review 38 (2012), 231-248; Schorkopf, F.: Europas politische Verfasstheit im Lichte des Fiskalvertrages, in: ZSE 10 (2012), 1-29; spez. zur deutschen Verfassungsrechtslage Umsetzung in Deutschland Möllers, C./Reinhardt, J.: Verfassungsrechtliche Probleme bei der Umsetzung des Europäischen Fiskalvertrages, in: Juristenzeitung 67 (2012), 693-700.

21 Lorz, A./Sauer, H., Ersatzunionsrecht und Grundgesetz, in: Die öffentliche Verwaltung 65 (2012), 573582. 
hat. Der bestehende Stabilitäts- und Wachstumspakt wurde unter dem Eindruck der insgesamt rund 60 Verstöße seit seinem Inkrafttreten überarbeitet. Zudem hat die Union ein neues Verfahren bei makroökonomischen Ungleichgewichten eingeführt. Es geht dabei um volkswirtschaftliche Kennziffern der Mitgliedstaaten, wie u.a. Lohnstückkosten und Leistungsbilanzsalden, die von der Kommission erhoben und an Schwellenwerten gemessen werden. Im Zuge des Sechserpakets wurde auch das Europäische Semester ${ }^{22}$ in das Sekundärrecht aufgenommen, mit dem man einen Zeitplan für die mitgliedstaatliche Haushaltsplanung sowie die Koordinierung mit der europäischen Ebene aufstellte. Mit dem Inkrafttreten von zwei weiteren Verordnungen im Mai 2013, dem sog. Two Pack, wird die Haushaltsüberwachung für die Euro-Staaten, insbesondere für Staaten in einem Defizitverfahren, nochmals verfeinert. ${ }^{23}$ Den Einzelheiten dieser umfangreichen Sekundärrechtsänderung ${ }^{24}$ kann im Rahmen dieses Beitrages nicht weiter nachgegangen werden. Für den hier vertretenen Standpunkt von Interesse sind die Erfahrungen mit der Anwendung des neuen Sekundärrechts.

Diese deuten darauf hin, dass es der Wirtschafts- und Währungsunion nicht an Normativität, sondern an Durchsetzungskraft ihrer Vollzugsorgane mangelt. In den laufenden Verfahren bei einem übermäßigen Haushaltsdefizit, das der Europäische Rat im Juni 2013 abschloss, hatte die Kommission für die Mitgliedstaaten Spanien, Frankreich, Niederlande, Polen, Portugal und Slowenien vorgeschlagen, deren Korrekturfrist um weitere ein bis zwei Jahre zu verlängern. Die Fristverlängerung beruht auf Art. 3 Abs. 5 VO (EU) Nr. 1467/97. ${ }^{25}$ Danach kann einem Mitgliedstaat - ohne Verschärfung des Defizitverfahrens - eine längere

22 Rat der Europäischen Union: Presseerklärung v. 7.9.2010, 5.

23 VO (EG) Nr. 472/2013 des Europäischen Parlaments und des Rates v. 21.5.2013 über den Ausbau der wirtschafts- und haushaltspolitischen Überwachung von Mitgliedstaaten im Euro-Währungsgebiet, die von gravierenden Schwierigkeiten in Bezug auf ihre finanzielle Stabilität betroffen oder bedroht sind, AB1. 2013 L 140/1; VO (EU) Nr. 473/2013 des Europäischen Parlaments und des Rates v. 21.5.2013 über gemeinsame Bestimmungen für die Überwachung und Bewertung der Übersichten über die Haushaltsplanung und für die Gewährleistung der Korrektur übermäßiger Defizite der Mitgliedstaaten im Euro-Währungsgebiet, AB1. 2013 L 140/11.

24 Einen Überblick gibt das Jahresgutachten 2012/13 des Sachverständigenrates zur Begutachtung der gesamtwirtschaftlichen Entwicklung, November 2012, Anhang zum zweiten Kapitel, 117-149. Näher zu einer juristischen Würdigung Di Fabio, U.: Die Zukunft einer stabilen Wirtschafts- und Währungsunion, 2013; spez. zum Six Pack Antpöhler, C.: Emergenz der europäischen Wirtschaftsregierung, in: Zeitschrift für ausländisches öffentliches Recht und Völkerrecht 72 (2012), 353-393; Bast., J./Rödl, F.: Jenseits der Koordinierung?, in: Europäische Grundrechte-Zeitschrift 39 (2012), 269-278.

25 VO (EG) Nr. 1467/97 des Rates v. 7.7.1997 über die Beschleunigung und Klärung des Verfahrens bei einem übermäßigen Defizit, AB1. 1997 L 209/6, in der Fassung der VO (EU) Nr. 1177/2011 des Rates v. 8.11.2011, ABl. 2011 L 306/33. 
Frist zur Korrektur eines übermäßigen Defizits gesetzt werden, wenn (i) ein unerwartetes wirtschaftliches Ereignis mit sehr ungünstigen Auswirkungen auf den betroffenen Mitgliedstaat eingetreten ist, so dass die Korrekturfrist nicht mehr eingehalten werden kann, und (ii) der Mitgliedstaat wirksame Maßnahmen ergriffen hat, um der an ihn gerichteten Empfehlung oder Inverzugsetzung des Rates nachzukommen, d.h. er muss ,,in Einklang mit der Empfehlung des Rates gehandelt haben. ${ }^{\text {26 }}$ Mit anderen Worten: Hat sich der Mitgliedstaat bemüht, durch politische Maßnahmen den negativen Saldo zu verändern und ist es zu „unerwarteten Ereignissen“ gekommen, kann die Frist verlängert werden.

Bei einer politischen Betrachtung dieses Vorgangs wird deutlich, dass damit zugleich der Reformdruck, der für wirtschafts- und sozialpolitische Rahmenänderungen notwendig ist, von dem betroffenen Mitgliedstaat genommen wird. Exemplarisch auf den wichtigen Fall Frankreichs angewendet, bedeutet die genannte Regelung für die Praxis eine Fristverlängerung bis zum Jahr $2015 .{ }^{27}$ So soll Frankreich nunmehr ein gesamtstaatliches Defizit von 3,9\% des BIP für 2013, 3,6 \% für 2014 und 2,8 \% für 2015 anstreben.

Der Rechtsrahmen der wirtschafts- und haushaltspolitischen Überwachung der Euro-Staaten wurde so umgebaut, dass der Schraubstock juristischer Rationalität nicht mehr entscheidend greifen kann. Die Europäische Kommission hat einen so weiten Beurteilungs- und Entscheidungsfreiraum erhalten, dass ihr der Vorwurf des rechtswidrigen Handelns kaum noch zu machen ist und sie allein nach politischen Maßstäben - wie auch der Europäische Rat - handeln kann. ${ }^{28}$ Das Recht steuert, aber es zwingt nicht.

Der zitierte Fall ist neben denen von Spanien und Slowenien von besonderer Bedeutung, ${ }^{29}$ weil in der Staatsschuldenkrise allgemein die Meinung herrscht,

26 Die Erklärung folgt in Duktus und Inhalt der Selbstdarstellung der Kommission, MEMO/13/463 v. 29.5.2013.

27 Der Vollständigkeit halber muss erwähnt werden, dass Art. 3 Abs. 5 VO (EU) 1467/97, op. cit. (Fn. 25) im Regelfall die Fristverlängerung um ein Jahr vorsieht, die Verdopplung der Frist müsste also eigens begründet werden.

28 Die Kommission hat, das muss ergänzt werden, in ihren länderspezifischen Empfehlungen durchaus konkrete Hinweise gegeben, welche Strukturreformen von Frankreich ergriffen werden sollten, um seine makroökonomischen Ungleichgewichte abzubauen; s. den Vorschlag für eine Empfehlung des Rates zum nationalen Reformprogramm Frankreichs 2013 mit einer Stellungnahme des Rates zum Stabilitätsprogramm Frankreichs für die Jahre 2012 bis 2017, KOM (2013) 360 v. 29.5.2013.

29 Das Kommissionshandeln im Jahr 2012 bezogen auf die Euro-Staaten untersucht Adamski, D.: Europe's (misguided) Constitution of Economic Prosperity, in: Common Market Law Review 50 (2013), 47-86, u.a. mit der nicht unberechtigten Überschrift „,The macroeconomic constitution of mutual congratula- 
die Euro-Staaten hätten durch die fiskalpolitischen Maßnahmen der Mitgliedstaaten (,Rettungsschirm“) und die Geldpolitik der Europäischen Zentralbank lediglich Zeit für nationale Reformen erhalten. Der ertüchtigte Stabilitäts- und Wachstumspakt ermöglicht also nunmehr ein pragmatischeres Defizitverfahren im Einklang mit dem Sekundärrecht, das jedoch Sinn und Zweck der wirtschaftsund haushaltspolitischen Überwachung widerspricht: auf ein solides öffentliches Haushalten und eine wettbewerbsfähige Wirtschaft hinzuwirken. ${ }^{30}$

\section{Dissens über die Wirtschaftspolitik in der Union}

Die gegenwärtige Krise macht darauf aufmerksam, dass die im vorherigen Abschnitt diskutierte Schwäche der Kommission, das Unionsrecht durchzusetzen, eine tiefer reichende Ursache hat: einen prinzipiellen Dissens über die Wirtschaftspolitik in der Europäischen Union. ${ }^{31}$

In der Europäischen Union werden Güter nach dem Marktprinzip verteilt. Bürger und Unternehmen nehmen nach geografischen, persönlichen und leistungsmäßigen Kenngrößen an der dezentralen Koordination der Produktion und des Konsums handelbarer Güter teil. ${ }^{32}$ Ihr Erfolg richtet sich nach ihrer Wettbewerbsfähigkeit, d.h. der Fähigkeit, in der Konkurrenz rentabel zu bestehen. ${ }^{33}$ Die Entscheidung der Mitgliedstaaten Mitte der 1950er Jahre, einen Gemeinsamen

tions“, op. cit. 50; und bereits ders.: National Power Games and Structural Failures in the European Macroeconomic Governance, in: Common Market Law Review 49 (2012), 1319.

30 Dieses Beispiel stimmt insoweit skeptisch, ob die Problemlösung in der Wirtschafts- und Währungsunion stets einem regelgeleiteten Ansatz folgen sollte. Der Gedanke, den wirtschafts- und haushaltspolitischen Wandel in den Mitgliedstaaten durch den Abschluss von bilateralen Reformverträgen (,Vertragspartnerschaften“) zu befördern, könnte dazu führen, dass wertungsoffene Vertragsnormen die Illusion einer Rechtsbindung schaffen, die im Ergebnis wiederum die Normativität des Rechts in der Union beschädigt. Über Vertragspartnerschaften wird nachgedacht, um die die Verbindlichkeit etwa der länderspezifischen Empfehlungen, op. cit (Fn. 28), zu erhöhen; von den Empfehlungen des Jahres 2012 sollen nur 10\% umgesetzt worden sein, EurActiv, Meldung v. 1.7.2013: Merkel zur Euro-Wirtschaftspolitik: „,Bisher kein gemeinsames Verständnis “. Die Skepsis wird befördert durch die Ankündigung der Kommission v. 3.7.2013, öffentliche Investitionen aus der Erfassung struktureller Defizitvorgaben heraus zu rechnen, um eine ,wachstumsverträgliche Haushaltskonsolidierung“ anzustreben.

31 Die Reaktion des französischen Staatspräsidenten auf die länderspezifischen Empfehlungen der Kommission, op. cit. (Fn. 28), die von ihr im Rahmen des zitierten Überwachungsverfahrens ausgesprochen wurden, betont dies. Francois Hollande wird mit den Worten zitiert: „Die EU-Kommission kann uns nicht diktieren, was wir zu tun haben, sie kann nur sagen, dass Frankreich seinen Haushalt ausgleichen soll“, zitiert nach Reuters v. 30.5.2013.

32 Siehe zur Wettbewerbsdefinition Magen, S.: Wettbewerb als sozialer Mechanismus, in: Kirchhof/Korte/ Magen (Hg.), Öffentliches Wettbewerbsrecht, Kapitel 3, 2014 i.E.

33 Die folgenden Überlegungen sind angeregt durch Thimann, C.: Die Bedeutung der Wettbewerbsfähigkeit auf dem Weg aus der Krise im Eurogebiet, Manuskript 2013. 
Markt mit einem möglichst unverfälschten Wettbewerb zu errichten, ${ }^{34}$ war - und ist auch weiterhin ${ }^{35}$ - eine Entscheidung für die Güterallokation durch privates Handeln in einer Geldwirtschaft. Der Kontrast zu dieser Entscheidung wird schärfer durch einen Seitenblick auf die Landwirtschaft, die dem Marktprinzip bewusst entzogen wurde. Zwar hieß es bereits im EWG-Vertrag, dass der Gemeinsame Markt auch die Landwirtschaft und den Handel mit landwirtschaftlichen Erzeugnissen umfasse (Art. 38 EWGV-1958). Die ausführlichen Sonderregelungen haben bis in die Gegenwart jedoch einen eigenständigen Politikbereich geschaffen, in dem das Marktprinzip unter dem Primat hoheitlicher Verteilungslenkung steht. Bei dieser „Entmarktlichung“ (Dekommodifizierung) handelt es sich um den historischen Kompromiss zwischen Deutschland und Frankreich, der die Wettbewerbsordnung im Übrigen ermöglichte und eine Landwirtschaftsreform in der Gegenwart erschwert. ${ }^{36}$

Die Mitgliedstaaten sind in den allgemeinen Sektoren weiterhin für die Wirtschaftspolitik zuständig, sie entscheiden über die Rahmenbedingungen des Arbeitens, der sozialen Sicherung, den Leistungsbeginn und -umfang der Rentenversicherung, die Haushalts- und Fiskalpolitik und das Maß staatlicher Intervention in die Wirtschaft. ${ }^{37}$ Die Mitgliedstaaten entscheiden mit ihrer Wirtschaftspolitik (zusammen mit den Tarifparteien) über die Lohnfindung, zugleich über die Kostenstruktur ihrer Wirtschaftsakteure und damit über deren Wettbewerbsfähigkeit. ${ }^{38}$ Die Folgen unterschiedlicher Wirtschaftspolitiken für die Wettbewerbsfähigkeit ihre Volkswirtschaften konnten die Mitgliedstaaten bis

34 Art. 2 i.V.m. Art. 3 lit. f EWG-Vertrag (1957).

35 Dass der Vertrag von Lissabon das Bekenntnis zum „System, das den Wettbewerb vor Verfälschungen schützt“ in das kleingedruckte Primärrecht eines Protokolls verschoben hat, ändert an der Grundaussage nichts.

36 Götz, V.:, 50 Jahre Gemeinsame Agrarpolitik, in: Juristenzeitung 67 (2012), 53 (54), ähnlich ders.: Das Recht der Gemeinsamen Agrarpolitik - Kontinuität, Wandel, Systematik, in: Martinez (Hg.), Die Gemeinsame Agrarpolitik vor neuen Herausforderungen, 2012, 11 (12).

37 Art. 119 Abs. 1, 120 AEUV sowie ausdrücklich EuGH, op. cit. (Fn. 16), Rn. 64, 160. Siehe auch, bezogen auf die länderspezifischen Empfehlungen, op. cit. (28), Europäischer Rat: Schlussfolgerungen v. 27./28.6.2013, EUCO 104/2/13 REV 2, Ziff. 4: „Die Mitgliedstaaten werden den Empfehlungen nun bei ihren anstehenden Entscheidungen über Haushalt, Strukturreformen und beschäftigungs- und sozialpolitische Maßnahmen Rechnung tragen und dabei die volle nationale Eigenverantwortung fördern und den sozialen Dialog aufrechterhalten. Der Rat und die Kommission werden die Umsetzung der Empfehlungen genau verfolgen."

38 Bemerkenswert die These, dass die Euro-Staaten nicht an demselben Maßstab für Wettbewerbsfähigkeit, sondern an einem historisch-kulturell angepassten Konzept gemessen werden müssten, Delors, J.: Economic Governance in the European Union, in: Journal of Common Market Studies 51 (2013), 169 (176). 
zum Eintritt in die dritte Stufe der Wirtschafts- und Währungsunion insbesondere durch eine Wechselkursänderung steuern. Mit der Übertragung der ausschließlichen Zuständigkeit für die Währungspolitik auf die Union haben die EuroStaaten dieses Instrument bewusst aus der Hand gegeben.

Der Reformdruck auf den wirtschaftspolitischen Rahmen der Mitgliedstaaten wächst, weil die Wettbewerbsfähigkeit nun im Wesentlichen direkt über Preise und Kosten gesichert oder errungen werden muss. Die Krise legt offen, dass einige Mitgliedstaaten zu den erforderlichen Arbeitsmarkt-, Renten-, Bildungsund Sozialreformen bislang nicht bereit sind. Weiter erinnert dieser Zusammenhang daran, dass bereits die negativen Referenden zum Verfassungsvertrag in Frankreich und den Niederlanden unter anderem damit erklärt wurden, dass Teilen der Bevölkerung die „,soziale Kälte“ des „,neo-liberalen“ Integrationsprojektes missfalle und dass die Logik der europäischen Rechtssetzung und Verwaltung das tradierte Sozialgefüge der Menschen und damit die nationale Identität verändere. ${ }^{39}$ Die Europäische Union wird verdächtigt, einzelnen Gesellschaften vorschreiben zu wollen, wie sie zu leben haben. Es kommt zu dem mit statistischen Daten unterlegten Kurzschluss, die völkervertraglich vereinbarte Grundordnung des Binnenmarktes sei nicht neutral, sondern bevorzuge einzelne industriestarke Mitgliedstaaten, besonders die Bundesrepublik Deutschland.

Der Dissens über die Grundordnung des Binnenmarktes kann integrationstheoretisch unterschiedlich gedeutet werden - in den beiden folgenden Abschnitten wird einzelnen Argumenten nachgegangen. Im Zusammenhang der Wirtschaftspolitik ist an dieser Stelle noch anzumerken, dass der geschilderte Zusammenhang die Logik des Binnenmarktes in einem Augenblick in Frage stellt, in dem die europäische Integration eine am grünen Tisch erhoffte Dynamik praktisch erreicht. Die Krise drängt Arbeitnehmer dazu, verstärkt in anderen Mitgliedstaaten auch über Sprach- und Kulturgrenzen hinweg nach Beschäftigung zu suchen. Konsumenten kaufen die Produkte im Binnenmarkt nach ihren privaten Vorstellungen. Es entsteht der Raum ohne Binnengrenzen, in dem Personen, Waren, Dienstleistungen und Kapital frei verkehren können. ${ }^{40}$

Diese Dynamik wird jedoch auch so wahrgenommen, dass nur ein bestimmter Teil von Unionsbürgern die Freizügigkeit nutzt und dadurch die demografischen, wirtschaftlichen und sozialen Probleme des Heimatstaates noch vergrößert, dass

39 Europäische Kommission: Eurobarometer, The European Constitution: Post-referendum survey in France, Juni 2005.

40 Vgl. Art. 26 Abs. 2 AEUV. 
die Wirtschaft nur bestimmter Mitgliedstaaten überproportional vom grenzenlosen Konsum profitiert und dadurch die Einnahmen des Staates erhöht. Durch die empfundene Unwucht wird der Dissens verstärkt und es entsteht Desintegration. Es kommt zu neuen Debatten über Wohlstandstransfers und die gerechte Güterverteilung in der Europäischen Union.

\section{Wohlstandstransfer und Gleichheitsfürsorge im europäischen Binnenmarkt}

Die gegenwärtige Debatte in der und um die Europäische Union ist ein Ringen um Verteilungskonzeptionen. Es geht um Antworten auf die Frage, wie bestehende europäische Zuständigkeiten ergänzt und durch effektivere Mechanismen ersetzt werden können. Letztlich geht es im Europäischen Rat um die Errichtung einer europäischen distributiven Sozialstaatlichkeit, wobei die Ideenwelt dieses Konzepts weiterhin diffus ist.

Im Rahmen des europäischen Integrationsprozesses wurde seit Ende der 1950er Jahre versucht, die Güterverteilung außerhalb des distributiv Sozialen zu organisieren. Dabei achtete die Gemeinschaft darauf, neue Mitgliedstaaten nur dann zuzulassen, wenn diese auch ein materielles Wohlstandsniveau in relativer Nähe zum Durchschnitt der bisherigen Mitglieder aufwiesen. ${ }^{41}$ Ein europäischer Staat, der sich zu weit von der durchschnittlichen Leistungsfähigkeit der Mitgliedstaaten entfernt, neigt möglicherweise zu einem distributiven Ausgleich der Unterschiede.

Seitdem sich der Beitritt der zehn Transformationsstaaten abzeichnete, ist das Niveau der Ungleichheit in der Europäischen Union zu einem Problem geworden, in das auch Alt-Mitgliedstaaten mittlerweile einbezogen sind. Das Versprechen, durch die Mitgliedschaft im europäischen Club die Gesellschaft zu modernisieren, die Wirtschaft wettbewerbsfähig zu machen und einen Wohlstandspfad zu beschreiten, ist brüchig geworden. Selbst die erheblichen Finanztransfers in der Vergangenheit haben es nicht vermocht, den schwächeren Mitgliedstaaten zu einem dauerhaft eigenverantwortlichen und selbstbestimmten Status in der Wett-

41 Deutlich die Kopenhagen-Kriterien für den Beitritt, s. Europäischer Rat von Kopenhagen v. 21./22.6.1993, Schlussfolgerungen des Vorsitzes, Dok. SN 180/1/93, 13: Die Mitgliedschaft ,erfordert ferner eine funktionsfähige Marktwirtschaft sowie die Fähigkeit, dem Wettbewerbsdruck und den Marktkräften innerhalb der Union standzuhalten. Die Mitgliedschaft setzt außerdem voraus, daß die einzelnen Beitrittskandidaten die aus einer Mitgliedschaft erwachsenden Verpflichtungen übernehmen und sich auch die Ziele der politischen Union sowie der Wirtschafts- und Währungsunion zu eigen machen können.“ 
bewerbsordnung des Binnenmarktes zu verhelfen. ${ }^{42}$ Das wiederum erklärt die Konjunktur der Solidaritätsdebatte, die sich häufig an relativen Differenzen beim Einkommen und Vermögen entzündet. ${ }^{43}$

Im Grunde stehen diese Differenzen aber für den konzeptionellen Wechsel von einer Selbstbestimmungsfürsorge hin zu einem egalitären Grundverständnis im Sinne einer Gleichheitsfürsorge. ${ }^{44}$ Entsprechende Forderungen verbinden sich mit dem Demokratiediskurs, wonach eine echte europäische Demokratie eine Politik für die materiellen Bedürfnisse der Unionsbürger erfordere. ${ }^{45}$ Und, solange es diese „soziale Demokratie“ nicht gäbe, werde die Europäische Union durch das „Auseinanderdriften zwischen den verschiedenen nationalen Produktionsund Verteilungskulturen“ früher oder später ihrer selbst überdrüssig. ${ }^{46}$

Der europäische politische Rahmen soll stärker als bisher Solidarität zwischen den Mitgliedstaaten gewährleisten. Das marktorientierte Grundmodell der Integration, ,die Marktgerechtigkeit“, soll durch ,soziale Gerechtigkeit“" entsprechend angepasst oder sogar ersetzt werden. ${ }^{47}$ Es stellt sich die Frage, ob direkte Vertei-

42 Einen Versuch, „Ungleichheit“ nicht nur relativ, in den Mitgliedstaaten, sondern unionsweit zu quantifizieren unternimmt Bonesmo Fredriksen, K.: Income Inequality in the European Union, OECD Economics Department Working Papers, No. 952, 2012, 11 ff., mit Hinweis auf die negativen Effekte durch die Beitritte, op. cit., 17 ff.

43 Dass die empfundene Wohlstandsverteilung in der Europäischen Union nicht unbedingt den statistischen Daten entspricht, zeigen die Ergebnisse der vom Eurosystem durchgeführten Haushaltsbefragung zu Finanzen und Konsum (Household Finance and Consumption Survey - HFCS), vgl. die Zusammenfassung in Europäische Zentralbank, Monatsbericht, April 2013, 65-81; zur Relativierung der Ergebnisse durch Bundeskanzlerin Angela Merkel, siehe Interview mit der Bild-Zeitung v. 19.4.2013, zugänglich unter http://www.bundeskanzlerin.de/Content/DE/Interview/2013/04/2013-04-19-merkel-bild.html. Die Bundesbank hat die EZB-Studie ergänzt und unternimmt einen Erklärungsversuch für das kontraintuitive Ergebnis, Deutsche Bundesbank, Vermögen und Finanzen privater Haushalte in Deutschland, Monatsbericht Juni 2013, 25-51.

44 Vom Standpunkt der theoretischen Konzeption des Sozialstaats Kersting, W.: Philosophische Sozialstaatsbegründung, in: Goldschmidt/Wohlgemuth (Hg.), Die Zukunft der Sozialen Marktwirtschaft, 2004, 9 (21 f.); ders.: Von der Verteilungsgerechtigkeit zur politischen Solidarität - Probleme der Sozialstaatsbegründung, in: Hildemann (Hg.), Die Zukunft des Sozialen, 2002, 67 (74 ff.).

45 Goulard, S./Monti, M.: De la démocratie en Europe, 2012; dazu die Besprechung von v. Bogdandy, A.: in: FAZ v. 6.3.2013, 6 .

46 Somek, A.: Sozialpolitik in Europa: Von der Domestizierung zur Entwaffnung, in: Bast/Rödl (Hg.), Wohlfahrtsstaatlichkeit und soziale Demokratie in der Europäischen Union, Europarecht Beiheft 1/2013, 49 (68), unter Hinweis auf die Studie von Höpner, M./Schäfer, A.: Grenzen der Integration - wie die Intensivierung der Wirtschaftsintegration zur Gefahr für die politische Integration wird, in: integration 2010, 3-20.

47 So eine der Thesen aus dem kritischen Spätkapitalismus-Manifest von Streeck, W.: Gekaufte Zeit. Die vertagte Krise des demokratischen Kapitalismus, 2. Aufl., 2013, 236. 
lungsentscheidungen der Union rechtfertigbar sind und ob sie die Verteilungskonflikte institutionell bewältigen können. ${ }^{48}$

Die politische Forderung, in der europäischen Wertegemeinschaft über den Wert „Demokratie“ (Art. 2 Satz 1 EUV) knappe Güter mit Mehrheitsentscheid zu verteilen, ist indifferent gegenüber der Integrationsgeschichte. Die Krise der „Politik des leeren Stuhls“ entzündete sich am Übergang von der Einstimmigkeit zur Mehrheitsentscheidung im Rat. ${ }^{49}$ Es ging um Marktpreisregelungen im Landwirtschaftssektor, die Frankreich dazu veranlassten, insgesamt sechs Monate den Ratssitzungen fern zu bleiben. Der Luxemburger Kompromiss aus dem Jahr 1966 verdeutlichte, dass es Einwirkungsschwellen für die Mitgliedstaaten gibt, die nicht ohne ihre politische Zustimmung überschritten werden können. Es war für Frankreich von größter Bedeutung, dass der Landwirtschaftssektor aus dem Verteilungsprinzip „Markt“ herausgenommen wird. Auch wenn das geltende Unionsrecht substantielle Verteilungsentscheidungen vereinzelt schon heute ermöglicht, hat sich an dem Grundzusammenhang trotz der Jahrzehnte seit jener Krise nichts verändert: Ein Mehrheitsentscheid der EU-Organe über elementare Rahmenbedingungen der Wirtschafts-, Arbeits- und Sozialverfassung ist derzeit bestenfalls visionär. ${ }^{50}$ Der institutionelle Rahmen der Union wäre überfordert, die politischen Konflikte auszugleichen. Die ,sozial-politische Ideenwelt“ der Mitgliedstaaten, in deren Gesellschaften Umverteilung durch kulturelle Prägungen unterschiedlich wahrgenommen werden, ${ }^{51}$ dürfte sich kaum mit der Situation

48 Chalmers, D.: The European Redistributive State and a European Law of Struggle, in: European Law Journal 18 (2012), 667 (676 ff.).

49 Lahr, R.: Die Legende vom „Luxemburger Kompromiß“, Europa-Archiv 38 (1983), 223-232, der den „Kompromiss“ als agreement to disagree bezeichnet; für eine juristische Auslegung der Beschlüsse Mosler, H., National- und Gemeinschaftsinteressen im Verfahren des EWG-Ministerrats, Zeitschrift für ausländisches öffentliches Recht und Völkerrecht 26 (1966), 1-32; die Beschlüsse sind abgedruckt in: BullEWG 3-1966, 9; Europa-Archiv 21 (1966), D 85 ff.

50 Siehe insb. Art. 153 Abs. 5 AEUV, der die Anwendung der sozialpolitischen Kompetenz der Union auf das Arbeitsentgelt, das Koalitionsrecht und das Streikrecht ausdrücklich ausschließt; die nichtjuristischen Voraussetzungen „sich majorisieren zu lassen“ betont auch Huber, P. M.: op. cit. (Fn. 13), 229 (239). In diesem Partikularitätsanspruch sehen manche aber den Ansatzpunkt für die Problemlösung, siehe Bofinger, P./Habermas, J./Nida-Rümelin, J.: Einspruch gegen die Fassadendemokratie, in: FAZ v. 3.8.2012, S. 33; dies., Plus que jamais, 1'Europe, in: Le Monde v. 4.9.2012 (online-Ausgabe). In hellsichtiger Skepsis bereits Hellwig, F.: Die politische Tragweite der europäischen Wirtschaftsintegration, 1966, 14 ff. insb. 29.

51 Näher zu diesem Gedanken Dallinger, U.: Sozialstaatliche Umverteilung und ihre Akzeptanz im internationalen Vergleich: Eine Mehrebenenanalyse, in: Zeitschrift für Soziologie 37 (2008), 137-157; ähnlich die Aussagen zum ,idiosynkratischen Charakter wohlfahrtsstaatlicher Entwicklungen“ (Hervorhebung weggelassen) Kaufmann, F.-X.: Varianten des Wohlfahrtsstaats, 2003, 33 f. 
in den Entscheidungsgremien decken, die über Verteilungsfragen zu befinden hätten.

\section{Fiskalische Verteilung und politische Verantwortung}

Die jährliche Debatte um die Nettosalden der Mitgliedstaaten ist zu einem festen, aus Sicht der EU-Organe notorischen Ritual der europäischen Öffentlichkeiten geworden. ${ }^{52}$ In einem fünfjährigen Abstand aggregiert sich die Nettozahlerdebatte in den Verhandlungen über den so genannten mittelfristigen Finanzrahmen der Europäischen Union. Dieser fünf- bis siebenjährige Finanzplan soll sicherstellen, dass die Ausgaben der Union innerhalb der Grenzen ihrer Eigenmittel ,eine geordnete Entwicklung nehmen. "53 In der Finanz- und Staatsschuldenkrise steht die Entscheidung über den neuen mittelfristigen Finanzrahmen 2014-2020 an.

Die jüngste Debatte über den Finanzrahmen endete zunächst nach knapp zweijährigen Verhandlungen, Mitte März 2013. Der Europäische Rat einigte sich darauf, die Finanzmittel für tatsächliche Ausgaben auf 0,95\% des Bruttonationaleinkommens (BNE) der Europäischen Union, d.h. auf insgesamt 908.400 Mio. Euro für den sechsjährigen Planungszeitraum zu begrenzen. ${ }^{54}$ Die Staatsund Regierungschefs kürzten damit den Haushaltsansatz gegenüber den vorherigen Finanzrahmen und verweigerten der Kommission die geforderte fünfprozentige Haushaltssteigerung. ${ }^{55}$ Der Europäische Rat setzte sich mit diesem Standpunkt durch, obwohl das Europäische Parlament, das der erforderlichen Ratsverordnung zustimmen muss, seinen politischen Dissens mit dem Beschluss der Staats- und Regierungschefs bereits frühzeitig erklärt hatte. ${ }^{56}$ Zuletzt hatte

52 Exemplarisch die Studie von Willeke, F.-U.: Deutschland, Zahlmeister der EU, 2011.

53 Art. 312 Abs. 1 AEUV. Die neue Planungsperiode 2014-2020 ist der erste Anwendungsfall der neuen Rechtsgrundlage, die die Annahme des mittelfristigen Finanzrahmens als Verordnung vorsieht. Zu den Entstehungsbedingungen der mittelfristigen Finanzplanung als Versuch, die Nettopositionen der Mitgliedstaaten zu begrenzen Neheider, S.: Die Kompensationsfunktion der EU-Finanzen, 2010, 45 ff.; mit Blick auf die Verhandlungen Becker, P.: Lost in Stagnation, SWP-Studie S 18, August 2012.

54 Europäischer Rat, Schlussfolgerungen v. 8.2.2013, EUCO 37/13, Ziff. 6. Die Ausgabenobergrenze für die EU-28, der Beitritt Kroatiens wird insoweit vorweggenommen, wurde für den Zeitraum 2014-2020 wurde auf 959.988 Mio. EUR an Mitteln für Zahlungsverpflichtungen festgesetzt. Das entspricht 1\% des BNE der Europäischen Union.

55 Europäische Kommission: Mitteilung - Ein Haushalt für „Europe 2020“, KOM (2011) 500 endgültig, Ziff. 3 sowie Vorschlag für eine Verordnung des Rates zur Festlegung des mehrjährigen Finanzrahmens für die Jahre 2014-2020 v. 29.6.2011, KOM (2011) 398.

56 Europäisches Parlament: Pressemitteilung v. 11.2.2013, EU-Haushalt: Einigung des Europäischen Rates ist nur erster Schritt, unter Hinweis auf die Gemeinsame Erklärung der Vorsitzenden von vier 
die irische Ratspräsidentschaft eine Einigung verkündet, worauf der Berichterstatter im Europäischen Parlament sein Mandat für das Dossier niederlegte. ${ }^{57}$ Mit geringfügigen Änderungen in der Sache einigten sich die Präsidenten des Parlaments und der Kommission mit dem irischen Ratsvorsitz am Rande des Europäischen Rates Ende Juni 2013 dann doch auf dieses Ergebnis. ${ }^{58}$

Das Parlament und die Kommission sind sich einig, dass die gewachsene Zahl europäisierter Politikbereiche von einer ausreichenden Finanzausstattung getragen sein muss. Die Mitgliedstaaten haben schließlich mit dem Vertrag von Lissabon weitere Zuständigkeiten übertragen und bestehende Politiken vertieft. Über diesen Konnexitätsgedanken hinaus sind sich beide EU-Organe weiter einig, ,dass die Art und Weise der Entwicklung des Systems der Eigenmittel (...) einen unverhältnismäßigen Schwerpunkt auf die Nettosalden zwischen den Mitgliedstaaten legt und damit dem Grundsatz der Solidarität der EU widerspricht, das gemeinsame europäische Interesse verwässert und den europäischen Zusatznutzen weitgehend ignoriert". 59

Für das Parlament und die Kommission sind die Nettotransfers der falsche Bezugspunkt in der Debatte über die europäische Finanzausstattung. Die hohen Nettosalden seien möglicherweise hinzunehmen, ja notwendig, weil sie durch den Grundsatz der Solidarität zwischen den Mitgliedstaaten im gemeinsamen europäischen Interesse lägen und zudem einen europäischen Nutzen stifteten. ${ }^{60}$ Aus Sicht der Mitgliedstaaten ist das Maß des Nettotransfers von Finanzmitteln aus dem nationalen Haushalt in die Europäische Union aber eher eine Gebühr für die europäische Integration. Sie wird mit der monetären und ideellen Einnahme-

Fraktionen; Entschließung des Europäischen Parlaments v. 13.3.2013 zu den Schlussfolgerungen des Europäischen Rates im Rahmen seiner Tagung vom 7./8.2. betreffend den mehrjährigen Finanzrahmen, 2012/2803(RSP), P7_TA(2013)0078.

57 Der irische Ratsvorsitz und der Haushaltskommissar (MEMO/13/592) gaben am 19.6.2013 bekannt, dass sie sich mit dem Verhandlungsführer des Parlaments auf einen Kompromiss geeinigt hätten, wonach das Gesamtbudget für die sieben Jahre bei 960 Mrd. Euro liegen solle, mit der Möglichkeit des Übertrags nicht verbrauchter Mittel auf das nächste Haushaltsjahr und einer Revisionsklausel für Ende 2016.

58 Europäische Kommission: Elements of the political agreement on the European Union's future budget 2014-2020, MEMO/13/625 v. 27.6.2013, mit dem Wortlaut der endgültigen Fassung der Ratsverordnung.

59 Entschließung des Europäischen Parlaments v. 8.6.2011 zu der Investition in die Zukunft: ein neuer mehrjähriger Finanzrahmen (MFR) für ein wettbewerbsfähiges, nachhaltiges und inklusives Europa. Europäische Kommission, Mitteilung, op. cit. (Fn. 55), Ziff. 1.

60 Das Europäische Parlament zeigt in seiner Entschließung v. 13.3.2013, op. cit. (Fn. 56), Ziff. 11, eine Kompromisslinie in dieser Frage auf. 
seite saldiert. Dass dieser Ansatz zumindest legitim war, zeigt der so genannte Briten-Rabatt, der Großbritannien seit 1976 gut Zweidrittel der Nettozahlungen an den EU-Haushalt erspart, die nach der allgemeinen Berechnungsformel zu leisten wären. ${ }^{61}$ Die Mitgliedstaaten haben die britische Forderung über Jahrzehnte anerkannt und auf dem Europäischen Rat Ende Juni 2013 erneut bestätigt.

Hinter diesem Gegensatz steht die sich widersprechende Verantwortungsposition der handelnden Organe. Dabei gilt, dass weder das Europäische Parlament noch die Kommission die Einnahmeseite quellenbezogen zu verantworten haben. Es sind die Mitgliedstaaten, die im Rahmen ihres nationalen Politikraums die finanziellen Ressourcen für die europäische Integration bereitstellen und gegenüber der Öffentlichkeit rechtfertigen müssen - auch in Zeiten der Finanz- und Staatsschuldenkrise. Der Solidaritätsgedanke ist in seiner abstrakten Form für die Rechtfertigung nur begrenzt tragfähig, jedenfalls nicht ohne den Seitenblick auf die Rücklaufquote. ${ }^{62}$ Die Hauptlast der Rechtfertigung trägt eine Kosten-NutzenSaldierung. Innerhalb dieser Saldierung spielen Kompensationsgedanken eine Rolle, die durchaus einen Solidaritätsreflex haben. Die verlässliche Öffnung des nationalen Marktes für Waren, Dienstleitungen, Personen und Kapital aus den anderen Mitgliedstaaten beruht auf Gegenseitigkeit. Auch der schwächere Handelspartner muss die Chance bekommen, sich im Gemeinsamen Markt aus Mitteln der Regional- und Kohäsionspolitik zu entwickeln. Zu diesem Zweck sind unmittelbare Finanztransfers gerechtfertigt und werden auch akzeptiert.

Die Kommission und das Parlament haben diesen Zusammenhang der Nettosalden mit der Kosten-Nutzen-Rechnung erkannt. Sie hatten vorgeschlagen, dass sich die Europäische Union „mit einem guten Anteil“ aus eigenen Mitteln finanzieren soll. Zu diesem Zweck legte die Kommission im Jahr 2011 auch einen Beschlussentwurf für den Rat vor, indem sie ein neues Eigenmittelsystem skizzierte. Der sehr komplexe Vorschlag sah u.a. neue Eigenmittelkategorien vor:

61 Siehe Wartenweiler, R.: Ungleichgewichte in den EG-Finanzstrukturen, in: Europa-Archiv 35 (1980), 521 (524 ff.); die Regelung wurde durch den Europäischen Rat von Fontainebleau in das Eigenmittelsystem mit der Begründung übernommen, dass ,jedem Mitgliedstaat, der gemessen an seinem relativen Wohlstand eine zu große Haushaltslast trägt, zu gegebener Zeit Korrekturen zugestanden werden können.“, BullEG 6/1984, Ziff. 1; zur geltenden Regelung siehe Art. 4 des Beschlusses Nr. 2007/436/EG des Rates v. 7.6.2007 über das System der Eigenmittel der Europäischen Gemeinschaften, ABl. 2007 Nr. L 63/17; neben der Korrektur für Großbritannien erhalten Deutschland, die Niederlande, Österreich und Schweden einen Rabatt.

62 Dazu näher Schorkopf, F.: Nationale Verfassungsidentität und europäische Solidarität, in: Calliess (Hg.), In Vielfalt geeint - Wieviel europäische Solidarität? Wieviel national Identität, Tübingen 2013 (i.E.), zugänglich als Berliner Online-Beitrag zum Europarecht Nr. 81 (2012). 
eine Finanztransaktionssteuer und eine neue Mehrwertsteuer-Einnahme. Das Aufkommen aus beiden Steuern soll direkt in den EU-Haushalt fließen. ${ }^{63}$ Die Finanzierung der Europäischen Union würde also nicht mehr als Ausgabe in den mitgliedstaatlichen Haushalten gebucht, sondern würde aufgrund privater Aktivität von Bürgern und Unternehmen über Einnahmen erfolgen. ${ }^{64}$ Das geplante neue Eigenmittelsystem würde aber nicht allein das Konfliktpotential der Haushaltsverhandlungen verringern. Es veränderte auch das Junktim von Verteilung und Rechtfertigung. Denn mit einer europäischen Steuer legte sich die Union ein neues, hoch wirksames Verteilungsinstrument $\mathrm{zu}^{65}$

Ein solcher Ratsbeschluss, der einstimmig getroffen und ratifiziert werden muss, ${ }^{66}$ wäre sicherlich vom herrschenden Standardlegitimationsmodell noch gedeckt. Die jeweiligen Beitragssummen aus den Mitgliedstaaten, speziell die Nettosalden, wären jedoch für die Zeit nach einer Ratifikation des neuen Eigenmittelsystems den nationalen politischen Räumen entzogen. Der primäre Adressat des Einnahmeinteresses der Europäischen Union wäre der Bürger, nicht mehr der jeweilige Haushaltsgesetzgeber. ${ }^{67}$ Der Bürger stünde der Europäischen Union nunmehr unmittelbar gegenüber und müsste seinen Interessen über die parzellierte Öffentlichkeit Gehör verschaffen. Der politische Hebel, über Wahlen auf das parlamentarische Regierungssystem einzuwirken - wie er im Bundestags-

63 Europäische Kommission: Vorschlag für einen Beschluss des Rates über das Eigenmittelsystem der Europäischen Union, KOM (2011) 510 v. 29.6.2011. Aus den Vorschlägen wird nicht recht deutlich, ob die „Einnahmen“ das bisherige System vollständig, d.h. aufkommensneutral ersetzen sollen.

64 Europäische Kommission, op. cit. (Fn. 63), 1: „Wichtiger dürfte hingegen der Umstand sein, dass die Art der Finanzierung des EU-Haushalts - durch Beiträge der Mitgliedstaaten an die EU, die viele Politiker auf nationaler Ebene nur als Ausgaben ansehen - zwangsläufig eine Spannung bewirkt, die jegliche Debatte über den EU-Haushalt vergiftet. Die wachsende Zahl von Korrekturmechanismen ist nur ein Symptom für dieses Problem. Ein anderes ist der Druck, die Mittelzuweisungen für die Mitgliedstaaten vorab festzulegen.“

65 Der Briten-Rabatt wurde seinerzeit mit dem Hinweis gerechtfertigt, dass die hohen Beiträge aus dem britischen Mehrwertsteueraufkommen dem relativen Wohlstand in Großbritannien nicht entsprächen. Ein originärer Mehrwertsteueranteil der EU am nationalen Aufkommen würde ein erhebliches Aufkommen aus den wirtschaftlich starken Mitgliedstaaten generieren. Zur politischen Debatte um eine europäische Steuer Begg, I.: Eine EU-Steuer. Überfällige Reform oder föderalistische Fantasie?, FESStudie, Februar 2011, als Hintergrund die Studie für die GD Haushalt des Netherlands Bureau for Economic Policy Analysis (CPB) und Institute for Economic Research (IFO), Autoren: Begg, I./Enderlein, H./Le Cacheux, J./Mrak, M. : Financing of the European Union Budget, Juni 2008.

66 Art. 311 Abs. 3 AEUV; vgl. dazu BVerfGE 123, 267 (387), Ziff. 312 f. - Lissabon (2009), wonach ein entsprechender Beschluss unter Art. 23 Abs. 1 GG fällt.

67 Die mögliche quantitative Entlastung der mitgliedstaatlichen Finanzen in Zeiten der Haushaltskonsolidierung durch das neue Eigenmittelsystem wird ausdrücklich von der Kommission hervorgehoben, op. cit. (Fn. 63), 2 f. Kritisch mit finanzverfassungsrechtlichen Argumenten Waldhoff, C.: Steuerhoheit für die Europäische Union?, 2012, $13 \mathrm{f}$. 
wahlkampf und in dem Begriff der haushaltspolitischen Gesamtverantwortung sichtbar wird -, würde ihm aus der Hand genommen.

Solange sich Unionsbürger primär als Bürger eines politischen Teilraums sehen, als Bürger eines wohlhabenden Alt-Mitgliedstaats, eines aufstrebenden Transformationsstaates oder eines hilfsbedürftigen Programm-Mitgliedstaates, wird das Europäische Parlament die Rechtfertigungslast nicht tragen können. Das neue Eigenmittelsystem wird nämlich zu einer Umverteilung zwischen den politischen Teilräumen in Europa führen, ohne dass die jeweiligen Vertreter im weiterhin territorial radizierten Parlament dies verhindern oder substantiell beeinflussen könnten. Das Europäische Parlament repräsentiert jedoch weiterhin nur eine fiktive europäische politische Zugehörigkeit gegenüber den divergenten, aber realen Verteilungsinteressen. ${ }^{68}$ Zugleich müssen die Abgeordneten sich für die Ressourcenbeschaffung gegenüber den Unionsbürgern nicht politisch rechtfertigen. Sie könnten nicht für die Gesamtbelastung des Bürgers ,geradestehen“, obgleich sie darüber entscheiden, wie die Finanzmittel ausgegeben werden.

Die Verteilungszuständigkeiten entfernen sich vom Menschen, während der hoheitliche Zugriff auf die persönlichen Güter fortbesteht und sich sogar verstärkt. Der eingeschlagene Weg intergouvernementaler Finanzhilfe und - demnächst - verstärkter Transferzahlungen zwischen den Euro-Staaten ist eine Antwort auf dieses Problem.

\section{Leitbilder der Integration und ein europäisches Deutschland}

In der europäischen Integration hatten und haben historisch begründete Leitbilder eine tragende Funktion. Mit historischen Argumenten wird die europäische Integration an sich gerechtfertigt, ihre Idee begründet, eine sinngebende Erzählung gestiftet. Dieser Logos der Integration wiederum ist maßgebend für die Rechtsauslegung, denn ein geschichtsindifferentes Recht wird neben dem Wortlaut vor allem über den effet utile ausgelegt.

In der integrationsgeschichtlichen Literatur wurde vorgeschlagen, zwei Grundströmungen zu unterscheiden: ${ }^{69}$ Die eine, für die der deutsche Historiker Walter

68 Selbst im deutschen Bundesstaat, in dem sich ein politischer zentraler Raum seit 1867 entwickelt, sind die Verteilungseffekte weiterhin umstritten, wie die neuen Verfahren Bayerns und Hessens vor dem Bundesverfassungsgericht gegen den Länderfinanzausgleich zeigen; strukturell vergleichbar ist die Lage im belgischen Bundesstaat.

69 Die Unterscheidung folgt Loth, W.: Explaining European Integration, Journal of European Integration History 14 (2008), 9 (12 ff.). Eine anregende dreigliedrige Differenzierung in „Europe of States“, „Eu- 
Lipgens steht, denke vom Bürger. Die europäische Integration sei die Antwort auf die Jahrzehnte des Krieges und der Vernichtung. Mehr noch, die Überforderung des Einzelnen durch die technische wie die politisch-militärische Entwicklung zeige, dass der politische Raum des Nationalstaates zu klein für diese Herausforderungen sei. Etwas Größeres müsse an dessen Stelle treten, um der europäischen Kultur das seit Langem gesuchte Zuhause geben zu können. ${ }^{70}$ Die Denkströmung gilt der Überwindung des Nationalstaates, der Suche des Bürgers nach einem neuen Gehäuse. Sie hat das deutsche Integrationsbewusstsein besonders geprägt.

Für die andere Denkströmung steht der britische Historiker Alan Milward. ${ }^{71}$ Er prägte die These, dass die europäische Integration von den Nationalstaaten getragen sei, um die Wünsche ihrer Bürger nach Frieden und Wohlstand zu erfüllen. Im Wortsinn der These geht es um die Rettung des Nationalstaats (Rescue of the Nation State) - der Nationalstaat sei in Europa nach dem Ende des Zweiten Weltkriegs, losgelöst von Gewinnern und Verlierern, zwar weiter als Staat, aber eben nur noch als integrierter Staat denkbar. Dieser Denkansatz ist realistisch und ließe Integration dort enden, wo die ursprünglichen Wünsche erfüllt sind. Es geht also nicht darum, die Vereinigten Staaten von Europa zu gründen, um den Traum vom einigen Europa zu verwirklichen. Es geht darum, den Völkern ihren jeweiligen Weg zu Frieden, Freiheit und Wohlergehen im gemeinsamen politischen Raum Europa zu ebnen.

Mit dieser Unterscheidung ließe sich erklären, warum sich die Mitgliedstaaten auf die Wirtschafts- und Währungsunion in der institutionellen Architektur des Unionsvertrages von Maastricht eingelassen haben, trotz der frühen Erkenntnis, dass ein einheitlicher Währungsraum eine gemeinsame Wirtschaftspolitik - es wird auch von der Politischen Union gesprochen - erfordert: Entweder diese These trägt nicht und die Währungsunion ist erfolgreich, oder die vorhergesagten Probleme stellen sich ein. Dann ist die Krise eine Chance, neue substantielle Integrationsschritte zu setzen, für die es unter Normalbedingungen keine politi-

rope of Offices“ und „Europe of Citiziens“ unternimmt van Middelaar, L.: The Passage to Europe, 2013 (niederl. Orig. 2009).

70 Lipgens, W.: Die Anfänge der europäischen Einigungspolitik 1945-1950, Bd. I: 1945-1947, 1977; ders.: Der Zusammenschluß Westeuropas. Leitlinien für den historischen Unterricht, in: Geschichte in Wissenschaft und Unterricht 34 (1983), 345-372; ders. (Hg.): 45 Jahre Ringen um die Europäische Verfassung. Dokumente 1939-1984, 1986.

71 Milward, A. S.: The Rescue of the Nation State, 1992, 2. Aufl. 2000, und bereits ders.: The Reconstruction of Western Europe, 1984. 
sche Mehrheit gibt. Letzteres entbehrt nicht des Zynismus, denn die Währungsunion entpuppte sich als ein Experiment in einer währungsbasierten Eigentumsordnung.

Die gegenwärtige Krise, in der sich einige der vorhergesagten Probleme einer „hinkenden Währungsunion“ eingestellt haben, zeigt nun, dass es am politischen Konsens fehlt, die vermeintliche Chance auf ein europäisches Europa zu ergreifen. Der erwähnte Streit über den mittelfristigen Finanzrahmen der Union betont den Willen der Mitgliedstaaten, die Haushaltsgröße der Europäischen Union bei gut $1 \%$ des BNE der Mitgliedstaaten zu belassen. ${ }^{72}$ Für Fiskalkapazitäten, mit denen eine Verteilung im Sinne einer supranationalen Fiskalunion durch EUOrgane möglich würde, soll demnach kein Platz sein. Die Staats- und Regierungschefs im Europäischen Rat haben sich gegenüber Kommission und Parlament durchgesetzt. Mehr noch, die kritische Aufmerksamkeit, mit der die Geldpolitik des Eurosystems verfolgt wird, das exakte Nachrechnen des deutschen Anteils an den umfangreichen Finanzhilfemaßnahmen, die die Euro-Staaten im Rahmen intergouvernementaler Konstruktionen untereinander bereitstellen, die empörte Ablehnung auswärtiger Solidaritäts- und Transferansprüche, belegen die Skepsis in Deutschland gegenüber der Notwendigkeit eines großen Integrationssprunges.

Die Finanz- und Staatsschuldenkrise legt Bruchstellen offen und markiert den prekären Stand der kulturellen, politischen und wirtschaftlichen Integration in Europa. Die Mitglieder des Europäischen Rates sind sich nicht einig darüber, wie die Wirtschafts- und Währungsunion zu vollenden ist. ${ }^{73}$ Deutschland hat $\mathrm{zu}$ dieser Krise der Europäischen Union nicht nur dadurch beigetragen, dass es bei den Beitritten zur Währungsunion nicht so genau hinsah ${ }^{74}$ und 2003 die Regeln des Stabilitäts- und Wachstumspaktes - Hand in Hand mit Frankreich - missachtete. ${ }^{75}$ Seine gesellschaftlichen und politischen Eliten haben zu lange Zeit an idealisierten Integrationsleitbildern festgehalten, die zahlreiche Integrationsschritte negativ beeinflussten. Es wäre Einiges gewonnen, wenn die maßgebenden Ak-

72 In den Diskussionen im Delors-Ausschuss, der die Währungsunion vorbereitete, sei angenommen worden, dass der Unionshaushalt zum Zeitpunkt des Eintritts in die dritte Stufe ein Volumen von 3\% des gemeinsamen BIP haben würde, zit. nach James, H.: Making the European Monetary Union, 2012, 400 .

73 Zuletzt Europäischer Rat: Schlussfolgerungen v. 27./28.6.2013, EUCO 104/2/13 REV 2, Ziff. 4-16.

74 Loth, W: Kreativ, vor allem in Buchführung. Wie Athen in die Eurozone gelangte und Berlin seinen Widerstand aufgab, in: Frankfurter Allgemeine Sonntagszeitung v. 18.9.2011, Nr. 37, 11.

75 EuGH, Rs. C-27/04, Slg. 2004, I-6649 - Kommission/Rat und Interview mit Gerhard Schröder in: Passauer Neueste Nachrichten v. 9.9.2011 (online-Ausgabe). 
teure ihr romantisches Leitbild aufgäben, den Nationalstaat bewusst zu überwinden. Es geht schon lange nicht mehr darum, ,dies Mißtrauen, diese Furcht zu zerstreuen" vor einem deutschen Europa, das niemand anstrebt. ${ }^{76}$ In auswärtigen Debatten über Europa werden an Deutschland inzwischen große Erwartungen herangetragen. ${ }^{77}$ Es geht deshalb im besten Sinn um ein europäisches Deutschland. $^{78}$

76 So aber Beck, U.: Das deutsche Europa, 2012.

77 Zuletzt das Titeldossier „The reluctant Hegemon“, The Economist v. 15.6.2013. Zur deutschen Hegemoniedebatte bereits Schönberger, C.: Hegemon wider Willen. Zur Stellung Deutschlands in der Europäischen Union, in: Merkur (2012) 752, 1-8. In diesem Kontext auch die Monographie von Simms, B.: Europe - The Struggle for Supremacy, 2013, insb. $458 \mathrm{ff}$.

78 Mann, Th.: Ansprache vor Hamburger Studenten (1953), in ders.: Gesammelte Werke, An die gesittete Welt, hg. v. Peter de Mendelssohn, 1986, 809 (811). 